Int. J. Electrochem. Sci., 14 (2019) $7380-7388$

\title{
Novel Detection of Acrylamide by Electrochemiluminescence Sensor and Optical Imaging Analysis
}

\author{
Xin Yang ${ }^{1,+}$, Peng Pan ${ }^{1,+}$, Li Tu ${ }^{1,+}$, Zhenyu Liao ${ }^{2,3}$, Huimin Niu ${ }^{1}$, Chuanlai Zang ${ }^{1}$, Mingchen Li ${ }^{1}$, \\ Jun Liu ${ }^{1, *}$, Zhengchun Yang ${ }^{1, *}$, Yangyang $Q i^{1, *}$, Jun Wei ${ }^{1,4}$, Kwok Wei Shah ${ }^{5}$ \\ ${ }^{1}$ School of Electrical and Electronic Engineering, Advanced Materials and Printed Electronics Center, \\ Tianjin Key Laboratory of Film Electronic \& Communication Devices, Tianjin University of \\ Technology, Tianjin 300384, China \\ ${ }^{2}$ Pony Testing International Group, Tianjin 300110, China \\ ${ }^{3}$ Tianjin Food Safety Inspection Technology Institute, Tianjin 300308, China \\ ${ }^{4}$ Singapore Institute of Manufacturing Technology, Agency for Science, Technology and Research \\ (A*STAR),71 Nanyang Drive, 638075, Singapore \\ ${ }^{5}$ School of Design and Environment, Department of Building, National University of Singapore, 4 \\ Architecture Drive, 117566, Singapore \\ +These authors contributed equally to this work. \\ *E-mail: cloudlj@163.com, yangzhengchuntjut@163.com, qyyqyqwx@163.com
}

doi: $10.20964 / 2019.08 .31$

Received: 10 April 2019 / Accepted: 20 June 2019 / Published: 30 June 2019

Acrylamide, a carcinogen with debilitating effects on human health, was detected by electrochemiluminescence (ECL) measurement using $\mathrm{Ru}(\mathrm{bpy})_{3}{ }^{2+}$ as the luminophore. Under the optimum conditions, Pt electrode as the working electrode showed a superior linear detection in the range $5 \mu \mathrm{M}-10 \mathrm{mM}$, with a detection limit of $1.2 \mu \mathrm{M}(\mathrm{S} / \mathrm{N}=3)$. Moreover, a remote wireless camera was employed to collect the digital images of the ECL emission. These images were converted from RGB (Red, Green, Blue) model to HSV (Hue, Saturation, Value) model using the MATLAB software. It was found that Value (V) in the HSV model had a linear relationship with the logarithm of the acrylamide concentration, suggesting that acrylamide could be detected in levels of $5 \mu \mathrm{M}$ using optical imaging. The ECL method for detecting acrylamide was proved to be simple and convenient. This is expected to lead to the development of a novel acrylamide detection method for food safety and other fields.

Keywords: electrochemiluminescence; acrylamide detection; optical imaging 
(C) 2019 The Authors. Published by ESG (www.electrochemsci.org). This article is an open access article distributed under the terms and conditions of the Creative Commons Attribution license (http://creativecommons.org/licenses/by/4.0/). 